\title{
The cardiovascular benefits of empagliflozin: SGLT2-dependent and -independent effects
}

\author{
Roberto Vettor $^{1} \cdot$ Silvio E. Inzucchi ${ }^{2} \cdot$ Paola Fioretto $^{1}$
}

Received: 8 November 2016 / Accepted: 15 November 2016 / Published online: 11 January 2017

(C) Springer-Verlag Berlin Heidelberg 2017

Keywords Cardiovascular disease $\cdot$ Empagliflozin $\cdot$ Heart failure $\cdot$ Mitochondrial function $\cdot$ NHE $\cdot$ SGLT2 inhibitors . Type 2 diabetes
Abbreviations
$\left[\mathrm{Ca}^{2+}\right]_{\mathrm{c}} \quad$ Cytoplasmic $\mathrm{Ca}^{2+}$ concentration
$\left[\mathrm{Ca}^{2+}\right]_{\mathrm{m}}$ Mitochondrial $\mathrm{Ca}^{2+}$ concentration
$\mathrm{HFH} \quad$ Heart failure hospitalisation
$\left[\mathrm{Na}^{+}\right]_{\mathrm{c}} \quad$ Cytoplasmic $\mathrm{Na}^{+}$concentration
NCX $\quad \mathrm{Na}^{+} / \mathrm{Ca}^{2+}$ exchanger
NHE $\quad \mathrm{Na}^{+} / \mathrm{H}^{+}$exchanger
ROS Reactive oxygen species
SGLT Sodium/glucose co-transporter

In the EMPA-REG OUTCOME trial, therapy with the sodium/glucose co-transporter (SGLT) 2 inhibitor empagliflozin over just 2.6 years was associated with a $14 \%$ reduction in the risk of major cardiovascular events, driven by a marked and unexpected reduction in cardiovascular mortality $(38 \%)$ in patients with type 2 diabetes and established cardiovascular disease [1]. The drug also reduced the incidence of heart failure hospitalisation (HFH) by $35 \%$.

Roberto Vettor

roberto.vettor@unipd.it

Paola Fioretto

paola.fioretto@unipd.it

1 Internal Medicine 3, Department of Medicine, University of Padua, via Giustiniani 2, 35128 Padova, Italy

2 Section of Endocrinology, Yale University School of Medicine, New Haven, CT, USA
The magnitude of these benefits and their rapid emergence after only a few months from randomisation make it unlikely that the modest benefits on $\mathrm{HbA}_{1 \mathrm{c}}(-0.4 \%$ compared with placebo), body weight $(-2 \mathrm{~kg})$ and systolic/diastolic blood pressure $(4 / 2 \mathrm{mmHg})$ were responsible [1]. Moreover, the divergence in the HRs for non-fatal myocardial infarction (HR 0.87 [95\% CI 0.70, 1.09]) vs non-fatal stroke (HR 1.24 $[0.92,1.67])$ makes it unlikely that the benefits of empagliflozin involved classical effects on atherosclerosis. Accordingly, additional mechanisms and mediators need to be considered to better understand why empagliflozin had such important benefits in this trial [2-5].

Based on their mode of action, SGLT2 inhibitors induce natriuresis and osmotic diuresis, associated with significant reductions in systolic and diastolic blood pressure. The resultant decrease in plasma volume and both cardiac preload and afterload may have obvious benefits for the heart, particularly in patients with impaired left ventricular function. However, it should be noted that only $10 \%$ of trial participants had recognised heart failure at baseline [6]. In addition, in clinical trials, anti-hypertensive agents and, specifically, diuretics, have not been demonstrated to have such strong protective cardiovascular effects, particularly on cardiovascular death and HFH. Only a recent trial involving eplerenone in patients with heart failure (but not necessarily diabetes) has demonstrated a similarly rapid improvement in heart failure outcomes [7]. Thus, it is conceivable that empagliflozin's diuretic activity contributed at least partially to the improved cardiovascular outcomes observed in EMPA-REG OUTCOME via an improvement in haemodynamics, decreasing myocardial stretch and the risk of developing potentially lethal arrhythmias. Yet, the results of this trial remain surprising, leading investigators to propose other mediators.

As noted, additional factors that might explain the cardiovascular protective effects of empagliflozin include weight 
reduction, improvement in glycaemic control, and other possible metabolic effects. It remains unlikely that the small amount of weight loss attributable to the drug contributed to the rapid reduction in cardiovascular mortality. Similarly, the modest improvement in glycaemic control with empagliflozin is unlikely to be a key contributor. However, worthy of consideration are the downstream effects of chronic negative energy balance from glycosuria and the resultant relative 'catabolic state' induced by SGLT2 inhibition [3]. This has been linked to the small elevation in glucagon levels (in the fasting state the increase in plasma glucagon levels ranges from $10 \%$ to $20 \%[8,9]$ ), proposed to play a possible role in cardiovascular protection. While some experimental data support a cardioprotective effect of glucagon [10], only one study in humans, from more than four decades ago, demonstrated a benefit from glucagon infusion in patients with heart failure and cardiogenic shock [11]. SGLT2 inhibitor-induced stimulation of glucagon secretion with simultaneous decreases in insulin secretion favour lipolysis, which increases circulating concentrations of NEFA, requiring a greater amount of oxygen to generate the same amount of ATP compared with glucose [3]. Heart metabolic flexibility implies that during fasting conditions there is a preferential utilisation of NEFA (and ketone bodies) for ATP generation. However when plasma levels of glucose and insulin rise, the contribution of glucose utilisation to ATP production increases. SGLT2 inhibitors appear to shift whole-body metabolism from glucose to fat oxidation, thus reducing the respiratory quotient (RQ) and increasing myocardial oxygen demand. This, in turn, could potentially worsen myocardial ischaemia in patients with type 2 diabetes, particularly those with preexisting impaired ventricular function and/or coronary insufficiency. Thus, the increased myocardial fat oxidation caused by empagliflozin in the EMPA-REG OUTCOME study can certainly not explain the reduction in cardiovascular mortality associated with use of the drug [3].

Simultaneously to and, indeed, related to the increased glucagon and NEFA levels, SGLT2 inhibition also causes a modest, but persistent hyperketonaemia. The heart avidly and preferentially removes and uses ketone bodies and their oxidation may improve cardiac muscle work efficiency, acting at the mitochondrial level [3-5]. In the isolated working heart $\beta$-hydroxybutyrate increases cardiac work efficiency by $24 \%$ while decreasing oxygen consumption. In addition $\beta$-hydroxybutyrate has been shown to reduce oxidative stress, stimulate mitochondrial biogenesis and stabilise cell membrane potential, with potential suppression of arrhythmogenesis $[4,5]$. Heart metabolic regulation is tightly linked with its function. Cardiac work and various ion pumps, particularly the $\mathrm{Ca}^{2+}$ ATPase pump in the sarcoplasmic reticulum, depend strongly on ATP generation, which derives mostly from oxidative phosphorylation in the mitochondria. Therefore, impairment of this metabolism-function relationship is relevant to diseases that involve or may lead to heart failure [12].

Several experimental observations support the concept that mitochondria are both the target and the origin of major pathogenic pathways that lead to the progression of myocardial dysfunction [13]. Therefore, mitochondrial cytopathy in heart failure could be the basis of a therapeutic strategy to maintain mitochondrial integrity and improve the myocardial contractile function. Empagliflozin-induced glycosuria and energy loss could also be viewed as a model of energy wasting that could increase mitochondrial biogenesis, thus mimicking what happens with food restriction or exercise [14-16].

In addition to the above-mentioned metabolic and haemodynamic effects of empagliflozin, it is also plausible that some direct effects, not directly mediated by SGLT2 inhibition, are in part responsible for the demonstrated cardioprotective effects of the drug. In the current issue, Baartscheer and co-workers hypothesised that empagliflozin could have a direct cardiac effect through lowering myocardial cytoplasmic sodium $\left[\mathrm{Na}^{+}\right]_{\mathrm{c}}$ and calcium $\left[\mathrm{Ca}^{2+}\right]_{\mathrm{c}}$ and enhancing mitochondrial calcium $\left[\mathrm{Ca}^{2+}\right]_{\mathrm{m}}$, these effects being independent from SGLT2 inhibition itself and actually mediated via $\mathrm{Na}^{+} / \mathrm{H}^{+}$exchanger (NHE) activity [17] (illustrated in Fig. 1).

In heart failure, elevated cardiac $\left[\mathrm{Na}^{+}\right]_{\mathrm{c}},\left[\mathrm{Ca}^{2+}\right]_{\mathrm{c}}$ and decreased $\left[\mathrm{Ca}^{2+}\right]_{\mathrm{m}}$ have been demonstrated in animal models. In addition, an increased SGLT1 expression, which couples ion transport to energy substrate metabolism by taking up two $\mathrm{Na}^{+}$ions together with one glucose molecule, has been found in failing hearts in patients with type 2 diabetes compared with those of nondiabetic individuals [18]. Several lines of evidence suggest that the consequent high $\left[\mathrm{Na}^{+}\right]_{\mathrm{c}}$ may contribute not only to the development of heart failure but also to the risk of sudden cardiac death due to increased arrhythmogenesis. It has also been shown that SGLT-mediated $\mathrm{Na}^{+}$and glucose entry are negligible in myocytes from control rats, but significantly increased in models of type 2 diabetes, probably as a maladaptation to attenuate insulin-mediated glucose uptake and chronic hyperglycaemia [18].

Using fluorescent probes the authors have also found that an increase in extracellular glucose produced a rise in $\left[\mathrm{Na}^{+}\right]_{\mathrm{c}}$ and $\left[\mathrm{Ca}^{2+}\right]_{\mathrm{c}}$ due, at least in part, to SGLT1 upregulation and activity. Empagliflozin treatment directly inhibited the NHE, caused a reduction of $\left[\mathrm{Na}^{+}\right]_{\mathrm{c}}$ and $\left[\mathrm{Ca}^{2+}\right]_{\mathrm{c}}$, and increased $\left[\mathrm{Ca}^{2+}\right]_{\mathrm{m}}$. Since the empagliflozin concentrations used are well below the $\mathrm{IC}_{50}$ of SGLT1 for empagliflozin, the authors conclude that these effects are independent of SGLT1. Therefore, it seems unlikely that partial SGLT1 inhibition by empagliflozin can explain the beneficial effect of the drug on cardiovascular events. However, the current available data do not fully rule out a potential role of SGLT1 inhibition, given the above-mentioned upregulation of 


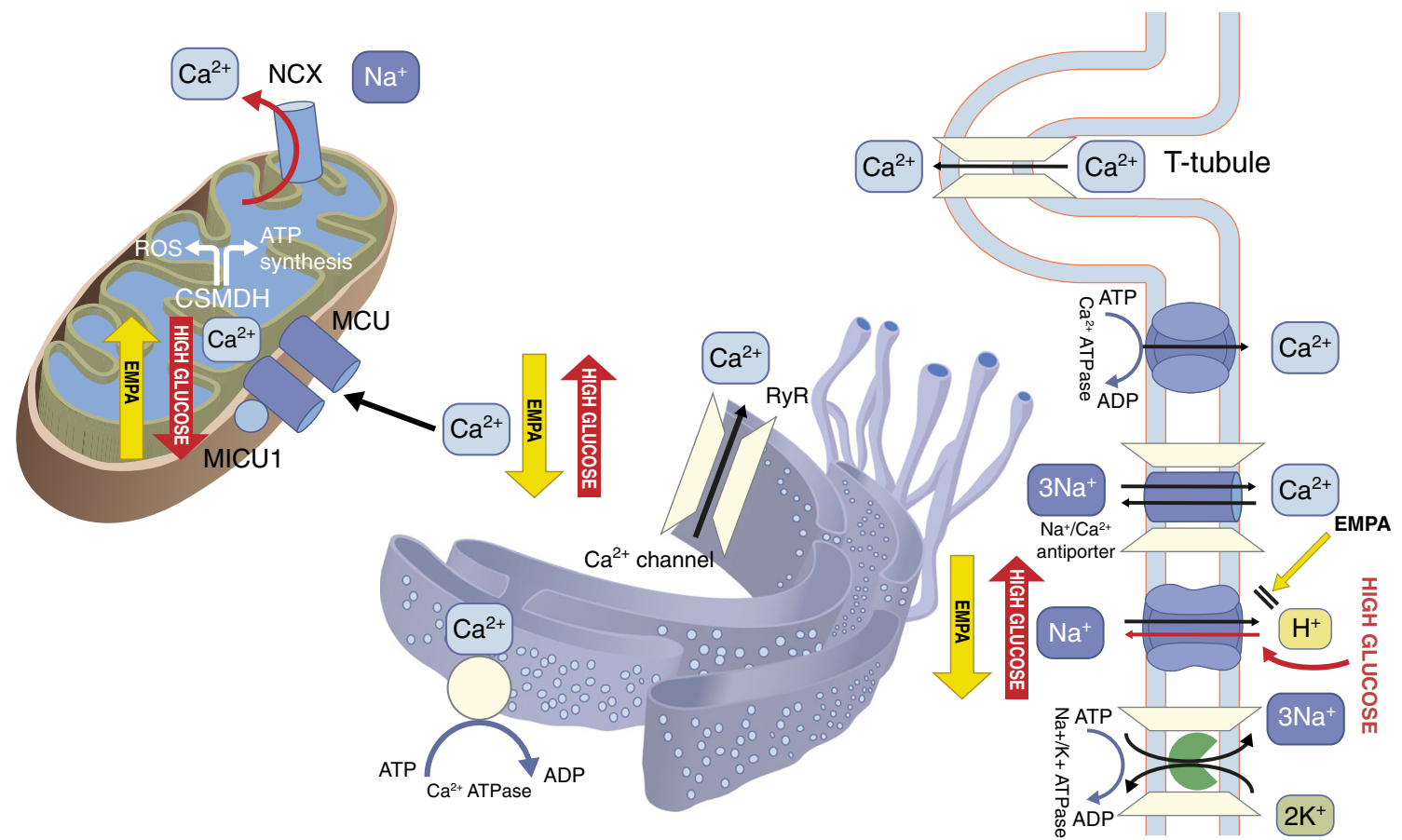

Fig. 1 Illustration of the NHE involvement in the failing myocardium during hyperglycaemia (red arrows). Activation of the exchanger occurs as a consequence of hyperglycaemia. The increased influx of $\mathrm{Na}^{+}$cannot be removed efficiently because of inhibition of $\mathrm{Na}^{+} / \mathrm{K}^{+}$ATPase. As a result, $\left[\mathrm{Na}^{+}\right]_{\mathrm{c}}$ levels increase, producing elevations in $\left[\mathrm{Ca}^{2+}\right]_{\mathrm{c}}$ levels via $\mathrm{Na}^{+} / \mathrm{Ca}^{2+}$ antiporter and $\left[\mathrm{Ca}^{2+}\right]_{\mathrm{m}}$ levels decrease, primarily via the inhibition of MCU. When an action potential is conducted, the calcium release from the sarcoplasmic reticulum via RyR induces accumulation of mitochondrial matrix calcium through $\mathrm{MCU}$, which is regulated by
MICU1, activates matrix calcium-dependent dehydrogenases, and then synthesis of intracellular ATP to support cardiac contraction. MCU is inhibited by hyperglycaemia and heart failure. EMPA (yellow arrows) treatment directly inhibits NHE, causing a reduction of $\left[\mathrm{Na}^{+}\right]_{\mathrm{c}}$ and $\left[\mathrm{Ca}^{2+}\right]_{\mathrm{c}}$, and an increase of $\left[\mathrm{Ca}^{2+}\right]_{\mathrm{m}}$, thus improving mitochondrial activity and ATP generation. CSMDH, mitochondrial dehydrogenase; EMPA, empagliflozin; MCU, mitochondrial $\mathrm{Ca}^{2+}$ uniporter; MICU1, mitochondrial calcium uptake 1 ; RyR, ryanodine receptor
SGLT1 in heart failure in type 2 diabetes. It is important also to keep in mind that the expression of SGLT2 and SGLT1 in the heart is still unclear: SGLT1 protein has been detected only in human heart capillaries while, in isolated mice cardiomyocytes, SGLT1 has been demonstrated within the T-tubules [17, 18].

The most important sodium transport mechanisms that may cause increased $\left[\mathrm{Na}^{+}\right]_{\mathrm{c}}$ are: $\mathrm{Na}^{+} / \mathrm{H}^{+}$exchanger (NHE)-1, $\mathrm{Na}^{+} /$ $\mathrm{HCO}_{3}$ cotransporter $(\mathrm{NBC}), \mathrm{Na}^{+} / \mathrm{K}^{+} / \mathrm{Cl}^{-}$cotransporter (NKCC), $\mathrm{Na}^{+}$channel, $\mathrm{Na}^{+} / \mathrm{K}^{+}$ATPase and $\mathrm{Na}^{+} / \mathrm{Ca}^{2+}$ exchanger (NCX). In this respect strong evidence exists that the detrimental effect of NHE-1 hyperactivity is due to the increase in $\left[\mathrm{Na}^{+}\right]_{\mathrm{c}}$ that leads to $\mathrm{Ca}^{2+}$ overload through the NCX, leading to myocardial dysfunction. Accordingly, inhibition of its hyperactivity appears to be a potential therapeutic strategy to prevent the harmful consequences of rises in $\left[\mathrm{Na}^{+}\right]_{\mathrm{c}}$ and $\left[\mathrm{Ca}^{2+}\right]_{\mathrm{c}}[19,20]$.

Calcium is an ambivalent signal: it is essential for the correct functioning of the cell, but may also become detrimental [21]. For any increase in cardiac workload, systolic $\left[\mathrm{Ca}^{2+}\right]_{\mathrm{c}}$ increases along with a parallel activation of mitochondrial metabolism, leading to an increased ATP formation. $\left[\mathrm{Ca}^{2+}\right]_{\mathrm{m}}$ plays an important role in heart energy production, coupling ATP demand. However, $\mathrm{Ca}^{2+}$ accumulation can impair mitochondrial function, leading to reduced ATP production and increased release of reactive oxygen species (ROS) contributing to heart failure (HF) $[21,22]$.

$\left[\mathrm{Ca}^{2+}\right]_{\mathrm{m}}$ signalling is critical for energy production but also for the activation of cell death pathways deeply implicated in the development of heart failure [21]. Mitochondrial NCX $(\mathrm{mNCX})$ is hypothesised to be the primary mechanism of $\left[\mathrm{Ca}^{2+}\right]_{\mathrm{m}}$ efflux. This antiporter pushes calcium out against its gradient, while bringing in a sodium ion to allow the heart to relax [19-21].

Thus, empagliflozin could lead to a reduction of $\left[\mathrm{Na}^{+}\right]_{\mathrm{c}}$ and $\left[\mathrm{Ca}^{2+}\right]_{\mathrm{c}}$, and an increase of $\left[\mathrm{Ca}^{2+}\right] \mathrm{m}$ through the inhibition of NHE activity. Conceivably, this effect, together with increased mitochondrial biogenesis and oxidation of ketone bodies, could lead to increased ATP generation with downstream benefits for cardiac viability and performance. Whether these effects are fully independent of SGLT2 inhibition remains an open matter for investigation.

In conclusion, pleiotropic actions of empagliflozin, both SGLT2-dependent and independent, involve the simultaneous 
modulation of multiple molecular and biochemical pathways and may not simply or exclusively be related to hyperglycaemia. Might any of these either alone or in concert explain the surprising benefits of this drug on cardiovascular outcomes as demonstrated in the EMPA-REG OUTCOME trial? Given the increased prevalence of and adverse prognosis from cardiovascular disease in patients with type 2 diabetes, it is important to determine the answer to this question. Studies like the one from Baartscheer et al should help in this quest.

Duality of interest SEI has received consultancy fees from Janssen; is on clinical trial steering committees for Boehringer Ingelheim; and is on the Clinical Trial Executive Committee at AstraZeneca. PF has received consultancy fees from AstraZeneca, Janssen and Boehringer Ingelheim. RV has no duality of interest in relation to this work.

Contribution statement All authors were responsible for drafting the article and revising it critically for important intellectual content. All authors approved the version to be published.

\section{References}

1. Zinman B, Wanner C, Lachin JM et al (2015) Empagliflozin, cardiovascular outcomes, and mortality in type 2 diabetes. N Engl $\mathrm{J}$ Med 373:2117-2128

2. Heerspink HJ, Perkins BA, Fitchett DH, Husain M, Cherney DZ (2016) Sodium glucose cotransporter 2 inhibitors in the treatment of diabetes mellitus: cardiovascular and kidney effects, potential mechanisms, and clinical applications. Circulation 134:752-772

3. Abdul-Ghani M, Del Prato S, Chilton R, DeFronzo RA (2016) SGLT2 inhibitors and cardiovascular risk: lessons learned from the EMPA-REG OUTCOME Study. Diabetes Care 39:717-725

4. Mudaliar S, Alloju S, Henry RR (2016) Can a shift in fuel energetics explain the beneficial cardiorenal outcomes in the EMPA-REG OUTCOME study? A unifying hypothesis. Diabetes Care 39:11151122

5. Ferrannini E, Mark M, Mayoux E (2016) CV protection in the EMPA-REG OUTCOME trial: a 'trifty substrate' hypothesis. Diabetes Care 39:1108-1114

6. Fitchett D, Zinman B, Wanner C et al (2016) Heart failure outcomes with empagliflozin in patients with type 2 diabetes at high cardiovascular risk: results of the EMPA-REG OUTCOME trial. Eur Heart J 37:1526-1534
7. Zannad F, McMurray JJ, Krum H et al (2011) Eplerenone in patients with systolic heart failure and mild symptoms. N Engl J Med 346:11-21

8. Ferrannini E, Muscelli E, Frascerra S et al (2014) Metabolic response to sodium-glucose cotrans- porter 2 inhibition in type 2 diabetic patients. J Clin Invest 124:499-508

9. Merovci A, Solis-Herrera C, Daniele G et al (2014) Dapagliflozin improves muscle insulin sensitivity but enhances endogenous glucose production. J Clin Invest 124:509-514

10. Murtagh JG, Binnion PF, Lal S, Hutchison KJ, Fletcher E (1970) Haemodynamic effects of glucagon. Br Heart J 32:307-315

11. Lvoff R, Wilcken DE (1972) Glucagon in heart failure and in cardiogenic shock. Experience in 50 patients. Circulation 45:534-542

12. Doenst T, Nguyen TD, Abel ED (2013) Cardiac metabolism in heart failure: implications beyond ATP production. Circ Res 113: 709-724

13. Rosca MG, Tandler B, Hoppel CL (2013) Mitochondria in cardiac hypertrophy and heart failure. J Mol Cell Cardiol 55:31-41

14. Vettor R, Valerio A, Ragni M et al (2014) Exercise training boosts eNOS-dependent mitochondrial biogenesis in mouse heart: role in adaptation of glucose metabolism. Am J Physiol Endocrinol Metab 306:E519-E528

15. Nisoli E, Clementi E, Carruba MO, Moncada S (2007) Defective mitochondrial biogenesis: a hallmark of the high cardiovascular risk in the metabolic syndrome? Circ Res 100:795-806

16. Marzetti E, Wohlgemuth SE, Anton SD, Bernabei R, Carter CS, Leeuwenburgh C (2009) Cellular mechanisms of cardioprotection by calorie restriction: state of the science and future perspectives. Clin Geriatr Med 25:715-732

17. Baartscheer A, Schumacher CA, Wüst RCI et al (2016) Empagliflozin decreases myocardial cytoplasmic $\mathrm{Na}^{+}$through inhibition of the cardiac $\mathrm{Na}^{+} / \mathrm{H}^{+}$exchanger in rats and rabbits. Diabetologia. doi:10.1007/s00125-016-4134-x

18. Lambert R, Srodulski S, Peng X, Margulies KB, Despa F, Despa S (2015) Intracellular $\mathrm{Na}^{+}$concentration $\left(\left[\mathrm{Na}^{+}\right]_{\mathrm{i}}\right)$ is elevated in diabetic hearts due to enhanced $\mathrm{Na}^{+}$-glucose cotransport. J Am Heart Assoc 4:e002183

19. Maack C, Cortassa S, Aon MA, Ganesan AN, Liu T, O'Rourke B (2006) Elevated cytosolic $\mathrm{Na}^{+}$decreases mitochondrial $\mathrm{Ca}^{2+}$ uptake during excitation-contraction coupling and impairs energetic adaptation in cardiac myocytes. Circ Res 99:172-182

20. Cingolani HE, Ennis IL (2007) Sodium-hydrogen exchanger, cardiac overload, and myocardial hypertrophy. Circulation 115:1090 1100

21. Griffiths EJ, Balaska D, Cheng WH (2010) The ups and downs of mitochondrial calcium signalling in the heart. Biochim Biophys Acta 1797:856-864

22. Rizzuto R, De Stefani D, Raffaello A, Mammucari C (2012) Mitochondria as sensors and regulators of calcium signalling. Nat Rev Mol Cell Biol 13:566-578 